\title{
Ultrasound Image Despeckling using Local Binary Pattern Weighted Linear Filtering
}

\author{
Simily Joseph, Kannan Balakrishnan \\ Digital Image Processing Lab, Dept. of Computer Applications, Cochin University of Science and Technology, Kerala, \\ India \\ E-mail: \{simily.joseph, mullayilkannan\}@gmail.com
}

M.R. Balachandran Nair

Ernakulam Scan Center, Kerala, India

E-mail:mrbala@gmail.com

Reji Rajan Varghese

Dept. of Biomedical Engineering, Co operative Medical College, Kerala, India E-mail:drrrvarghese@gmail.com

\begin{abstract}
Speckle noise formed as a result of the coherent nature of ultrasound imaging affects the lesion detectability. We have proposed a new weighted linear filtering approach using Local Binary Patterns (LBP) for reducing the speckle noise in ultrasound images. The new filter achieves good results in reducing the noise without affecting the image content. The performance of the proposed filter has been compared with some of the commonly used denoising filters. The proposed filter outperforms the existing filters in terms of quantitative analysis and in edge preservation. The experimental analysis is done using various ultrasound images.
\end{abstract}

Index Terms - Computer Aided Diagnosis, Filtering, Local Binary Pattern, Speckle Noise, Ultrasound Imaging

\section{Introduction}

Medical Imaging technologies could make drastic changes to clinical diagnosis process. The power of digital media and the advances in Information Technology offers highly intelligent and sophisticated methods for diagnosis and treatment ${ }^{[1]}$. The history of biomedical imaging has its root in the discovery of $\mathrm{X}$ ray by Wilhelm Roentgen in 1885 . Over the past hundred years, the growth of technologies from X-ray to MRI, CT, PET, SPECT, Ultrasound etc. changed the traditional horizon of clinical medicine. The efficiency of such non-invasive imaging tools grow rapidly with the advances in Computer Science. The possibility to perform both diagnosis and therapeutic procedures increases the wide spread use of ultrasonography. Low cost, non-invasive nature, painless examination, portability and instant diagnosis make ultrasound imaging a most prevalent tool in medical industry.

Ultrasound machine uses high frequency sound waves to capture pictures. The coherent nature of ultrasound imaging, results in the formation of a mu ltip licative noise called speckle noise. Speckle noise appears as a granular pattern which varies depending upon the type of biological tissue. The interference of backscattered signals result in speckle noise and its apparent resolution is beyond the functionalities of the imaging system. Noise content is usually stronger than the microstructure of tissue parenchyma and reduces the visibility and masks the tissue under investigation. Therefore the main challenge in despeckling is to filter the noise content without affecting the microstructures and edges.

Despeckling can be done in two ways. It can apply directly on phase of RF signal ${ }^{[2]}$ and it can also be used as post processing techniques applied on images. Different methods like linear and non linear filtering, wavelet based despeckling etc. have been proposed to reduce the noise. Chinrungrueng et al. propose edge preserving noise reduction method using two dimensional Savitsky Golay filters which is based on the least square fitting of a polynomial function to image intensities [3], [4]. Though it takes less computation time than median filter the effect of noise reduction was same as that of median filter. A modified hybrid median filter and adaptive weighted median filter have been reported in the literature ${ }^{[5],[6]}$. Sivakumar et al. proposes an improved mean filter using fuzzy similarity ${ }^{[7]}$. A cumulative speckle reduction algorithm for spatial and diffusion filter and the modified anisotropic diffusion based on partial differential equation has been developed ${ }^{[8],[9]}$. Filtering using wavelet transformation, multi scale wavelet domain transformation, wavelet shrinkage, wavelet 
thresholding anisotropic diffusion etc. can be found in various studies ${ }^{[10]-[16]}$. Another method based on Ricardo reduces the split spectrum processing that occurs as a result of one dimensional narrow band filters ${ }^{[17]}$. Yang et al. ${ }^{[18]}$ proposes an edge preserving denoising method based on bilateral filter and Gaussian scale mixtures in shiftable complex directional pyramid domain. Bhadauria et.al proposes an adaptive fusion of curvelet transform and total variation for denoising medical images ${ }^{[19]}$.

The proposed system is tested with ultrasound placenta images and kidney images. Ultrasound placenta images of different grades are shown in Figure 1. Ultrasound is the best ideal tool to determine the fetal growth and position ${ }^{[20]}$. Noise suppression is very important to arrive at correct decision and to overcome the variability in diagnosis. Human interpretation and diagnosis becomes easy and accurate. The novelty of the proposed work is the introduction of local binary patterns to noise reduction process. No despeckling algorithm has been reported in the literature using the LBP approach.

The rest of the paper is organized as follows. Section 2 describes the speckle noise in ultrasound images and different noise reduction algorithms that are considered in this study. The proposed algorithm is also given. In section 3 result analysis using ultrasound images and discussion are presented. Conclusion and future work are given in the final section.

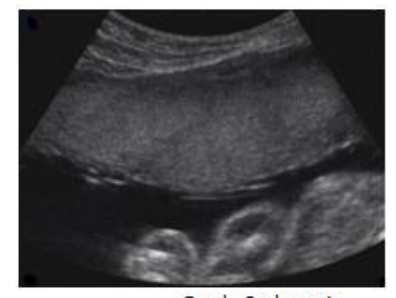

Grade 0 placenta

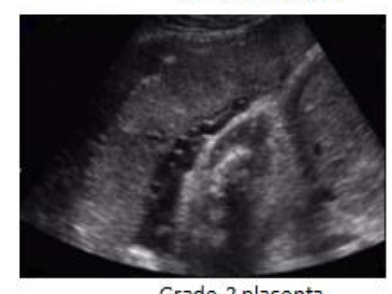

Grade 2 placenta

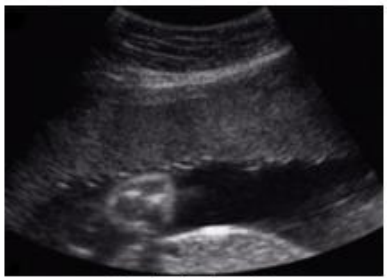

Grade 1 placenta

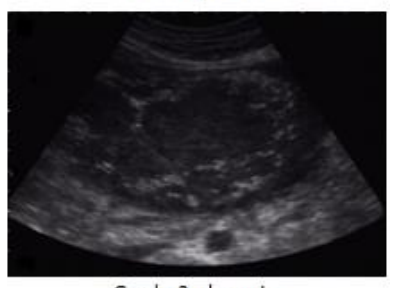

Grade 3 placenta
Fig. 1: Sample images of ultrasound placenta in different grades

\section{Speckle Noise in Ultrasound Imaging and Despeckling Methods}

Speckle is a form of multiplicative noise that affects the quality of ultrasound images. In ultrasound imaging the tissue under examination is a sound absorbing medium containing scatters. The inhomogenity of the tissue and the small size of image detail than the wavelength of the ultrasound results in the scattering of signals and lead to the formation of a granular pattern called speckle noise ${ }^{[21]}$. Multiple examination of the same object under different machine condition forms identical speckle patterns. Speckle patterns generally changes with the change in machine condition. There exists no relation between the speckle and texture pattern which depicts the actual histological structure of object. The output of ultrasound signal can be modeled as,

$$
g(x, y)=f(x, y) n(x, y)+\eta(x, y)
$$

Where $g(x, y)$ is the noisy pixel, $f(x, y)$ is the noise free pixel, $n(x, y)$ and $\eta(x, y)$ represents the multiplicative and additive noise respectively, $x$ and $y$ are the indices of the spatial locations which belong to the $2 \mathrm{D}$ space of real numbers. The effect of additive noise is less than that of multiplicative noise, therefore the above equation after logarithmic compression we can write as the sum of noise free pixel and noise component.

$$
g(x, y)=f(x, y)+n(x, y)
$$

Better image quality helps in easy and accurate diagnostic decision making. The widespread use of ultrasound imaging necessitates the need for developing despeckling filters for reducing noise. The performance of the proposed filter is compared with some of the existing filters such as mean filter, median filter, diffusion filter and wiener filter.

\subsection{Mean Filter}

Conventional mean filter replaces the value of every pixel in the image by the mean of the intensity level defined over a neighborhood. As a result sharp transitions in intensity get reduced. Mean filters fail to preserve edges and small details in an image. The basic linear filtering operation can be re presented as,

$$
f(x, y)=g^{-}+w(x, y)\left(g(x, y)-g^{-}\right)
$$

Where $g^{-}$is the local mean value of a region surrounding and including the pixel $g(x, y)$, $w(x, y)$ is the weight with values in the range $[0,1]$. The image obtained will be blurred.

\subsection{Wiener Filter}

Wiener filter is more data driven and relies on the homogeneous appearance of the image. It is developed based on the assumption that the image properties and the characteristics of noise are same over different regions. This is not true in practical situation. Therefore adaptive wiener filtering has been developed. Wiener filter also uses local statistics in a moving window. The term $w(x, y)$ in equation 3 becomes 


$$
w(x, y)=\left(\sigma^{2}-\sigma_{n}^{2}\right) / \sigma^{2} .
$$

Where $\sigma^{2}$ and $\sigma_{n}^{2}$ represent the variance in the moving window and the variance of noise in the image. In frequency domain wiener filter tends to weaken low energy components which give more information regarding image detail. In the power spectrum, the noise and image content show similar properties ${ }^{[22]}$.

\subsection{Diffusion Filter}

Perona and Malik [23] proposed a PDE (Partial differential Equation) based speckle noise reduction method using the concept of scale space. In scale space representation the original image is embedded into a family of more simpler and global representation where semantic information can be extracted easily. They tried to reduce the diffusivity of edge like locations in which a transition in intensity level occurs. The diffusion coefficient is adjusted to give more priority to intraregional smoothing than interregional smoothing. Perona Malik diffusion is based on the flowing equation.

$$
\partial_{t} u=\operatorname{div}\left(g\left(\left|\nabla_{u}\right|^{2}\right) \nabla_{u}\right.
$$

$$
\text { The diffusivity } g\left(s^{2}\right)=\frac{1}{1+s^{2} / \lambda^{2}} \quad(\lambda \succ 0)
$$

The disadvantage of diffusion filtering is the correspondence problem which occurs mainly due to the dislocation of edge from finer to coarser region. Also there exists the chance of losing some relevant information while smoothing.

\subsection{Median Filter}

Median filter is a non linear order static filter that replaces the middle pixel in the neighborhood window with the median value ${ }^{[7]}$. Generally an odd number is chosen as the size of the neighborhood so that a well defined center value exists. If the neighborhood region of a median filter contains an edge, it preserves the edge under the following conditions: the edge is straight within the neighborhood region, the signal difference of the two regions incident to the edge exceeds the noise amplitude and the signal is locally constant within each of the two regions ${ }^{[24]}$. Its performance is poor when the number of noise pixels is greater than or equal to half the number of pixels in the neighborhood.

\subsection{Proposed Local Binary Pattern Linear Filter}

LBP is a gray scale invariant that describes local primitives such as curved edges, points, spot, flat areas etc. ${ }^{[25]}$. LBP methodology has contributed a vital ro le in texture analysis. It is widely used in different computer vision problems such as face recognition, motion analysis, medical image analysis, finger print recognition, palm print recognition, vessel extraction of conjunctiva images etc. ${ }^{[2]-[31]}$. Over the past ten years different variant to LBP such as uniform patterns, dominant local binary pattern, combination of Gabor and LBP, center symmetric local binary pattern etc. has been reported in the literature ${ }^{[32]-[35]}$. To generate LBP code for a neighborhood, the weight assigned to each pixel is multiplied with a numerical threshold. The process is repeated for a set of circular samples. Figure 2 shows the process of LBP code generation for a circular neighborhood.

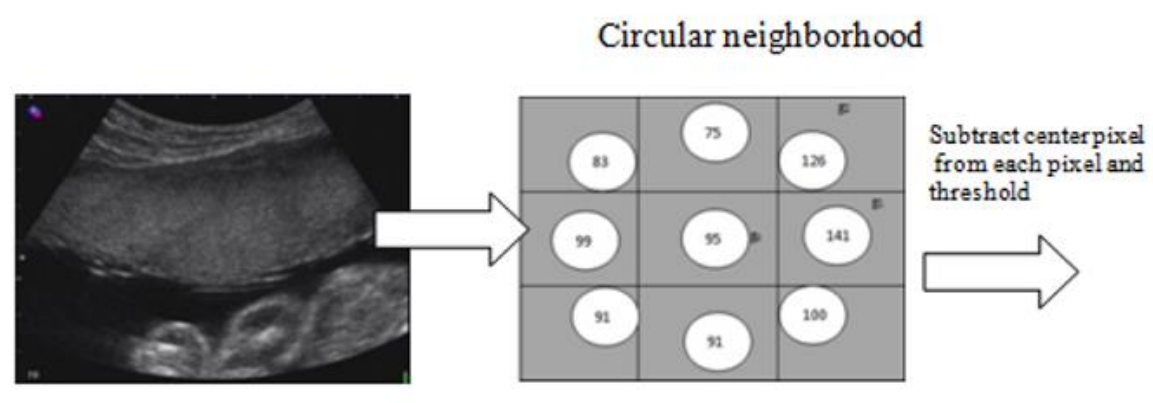

Binary code

LBP

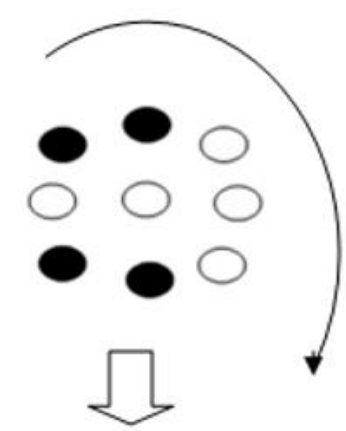

00111001

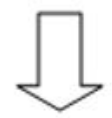

57

Fig. 2: Sample images of ultrasound placenta in different grades 
Texture over a neighborhood of pixels can be defined as the joint distribution of the gray value of a central pixel of the neighborhood say $\mathrm{g}_{\mathrm{c}}$ and gray value of circular pixels located at distance $P$.

$$
T=t\left(g_{c}, g_{0}, g_{1, \cdots . g_{p-1}}\right)
$$

The local texture pattern of a neighborhood can be obtained from the difference of central pixels and each pixel in the neighborhood. As the differences are independent this joint distribution can be factorized:

$$
T \approx t\left(g_{c}\right) t\left(g_{0}-g_{c}, \ldots, g_{p-1}-g_{c}\right)
$$

To make this invariant against all transformations the signs of the difference are also considered and the overall luminance $t\left(g_{c}\right)$ is ignored as it does not contribute anything to texture analysis.

$$
\begin{gathered}
T \approx t\left(s\left(g_{0}-g_{c}\right), \ldots . s\left(g_{p-1}-g_{c}\right)\right) \\
s(x)=\left\{\begin{array}{l}
1 x \geq 0 \\
0 x<0
\end{array}\right.
\end{gathered}
$$

By assigning weight, this difference is converted to a Local Binary Pattern Code which is equivalent to the local texture. The following expression will generate $2^{\mathrm{p}}$ LBP values for a neighborhood of pixels located at distance $\mathrm{p}$.

$$
\operatorname{LBP}_{P, R}\left(x_{c}, y_{c}\right)=\sum_{p=0}^{P-1} s\left(g_{p}-g_{c}\right) 2^{p}
$$

Where $g_{c}$ is the gray value of a central pixel of the neighborhood. The process is repeated for a set of circular samples. The histogram of the LBP codes is widely used as texture descriptor. To reduce the size of the descriptors only uniform patterns - binary patterns that occurs more frequently than others are considered. These values are normalized and used for the filtering operation. The proposed approach uses the local binary patterns as the coefficient of filtering. The proposed method follows a weighted linear filtering approach. It replaces the conventional local statistics in weighted linear filtering with local binary pattern approach. This method uses LBP to calculate the weight in the linear filter of the form:

$$
f(x, y)=g^{-}+w(x, y)\left(g(x, y)-g^{-}\right)
$$

\section{Results and Discussion}

The ultrasound imaging was carried out using Sonoline G50 ultrasound system with frequency ranging from 3.5 to $5 \mathrm{MHz}$. Machine settings were optimized to ensure that, the images taken are good in quality. The examinations were conducted transabdominally by an experienced radiologist. The despeckling process is carried out on a personal computer with Intel core 2 Duo process or of $2.93 \mathrm{GHz}$ speed with 4.00 GB RAM. The algorithm is implemented using Matlab 7 with Windows 7 as operating system. Initially, the well known median filtering has been applied to make the ultrasound image noise free. To the noise free image, speckle noise of zero mean and variance 0.2 are added. Then the original image is reconstructed using despeckling filters. Figure 3 shows the example of a noisy image.

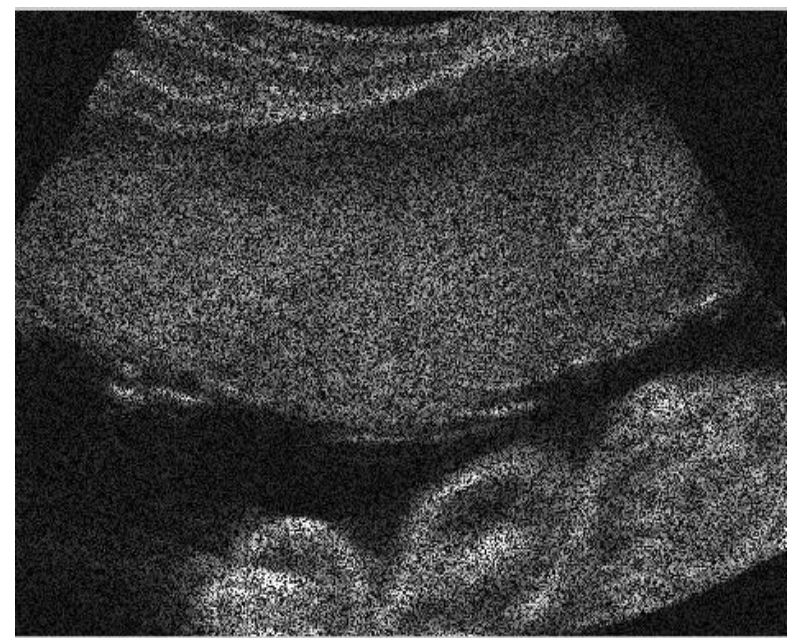

Fig. 3: ultrasound image corrupted with speckle noise

We compare the performance of the proposed method with some of the conventional approaches in speckle reduction: diffusion filtering, mean filtering and wiener filtering. The quantitative measurements used for performance evaluation are Mean Squared Error (MSE), Root Mean Squared Error (RMSE), Signal to noise Ratio (SNR), Peak Signal to noise Ratio (PSNR) and Structural Similarity Index (SSIN). The performance of the system is also evaluated using one of the best qualitative measurements - Universal Quality Index (UQI). It models any distortions in image as a combination of three factors such as loss of correlation, luminance distortion and contrast distortion. The following equations are used for computing these measures.

$$
M S E=\frac{1}{M N} \sum_{i=1}^{M} \sum_{j=1}^{N}(g(x, y)-f(x, y))^{2}
$$

$$
R M S E=\sqrt{\frac{1}{M N} \sum_{i=1}^{M} \sum_{j=1}^{N}(g(x, y)-f(x, y))^{2}}
$$




$$
S N R=10 \log _{10} \frac{\sum_{i=1}^{M} \sum_{j=1}^{N}\left(g(x, y)^{2}-f(x, y)^{2}\right)}{\sum_{i=1}^{M} \sum_{j=1}^{N}(g(x, y)-f(x, y))^{2}}
$$

$$
P S N R=-10 \log _{10} \frac{M S E}{g_{\text {max }}^{2}}
$$

$$
\begin{aligned}
\operatorname{SSIN}= & \frac{\left(2 g^{-} f^{-}+c_{1}\right)\left(2 \sigma_{g f}+c_{2}\right)}{\left(g^{-^{2}}+f^{-^{2}}+c_{1}\right)\left(\sigma_{g}^{2}+\sigma_{f}^{2}+c_{2}\right)}, \\
& -1<\operatorname{SSIN}<1
\end{aligned}
$$

$$
\begin{aligned}
U Q I= & \frac{\sigma_{g f}}{\sigma_{f} \sigma_{g}} \frac{2 f^{-} g^{-}}{\left(f^{-}\right)^{2}+\left(g^{-}\right)^{2}} \frac{2 \sigma_{f} \sigma_{g}}{\sigma_{f}^{2}+\sigma_{g}^{2}}, \\
& -1<U Q I<1
\end{aligned}
$$

Where $g(x, y)$ is the noisy pixel, $f(x, y)$ is the noise free pixel, $g^{-}$and $f^{-}$are the local mean value of a region in the noise free and noisy image, ${ }^{c}{ }_{1}$ and $c_{2}$ represents the dynamic range of ultrasound images ; $\sigma_{g} \sigma_{f}$ represents the standard deviations of original despeckled values of the analysis window and $\sigma_{g f}$ represents the covariance between original and despeckled windows.

The results in Table 1 clearly demonstrate that the proposed method outperforms the other filters. The edge map of the proposed filter shows high similarity to edge map of original image than the other filters which indicate the superior performance of the proposed method in edge preservation. Figure 4 shows the horizontal intensity profile of a scan line passing through the centre of the original image and despeckled image using different filters such as diffusion, mean, wiener and proposed LBP filter. We can observe a close similarity between the intensity profile of the proposed

\begin{tabular}{|c|c|c|c|c|c|c|}
\hline \multicolumn{7}{|c|}{ Grade 0 Placenta } \\
\hline & MSE & RMSE & SNR & PSNR & SSIM & UQI \\
\hline diffusion filter & 30.0673 & 5.4834 & 24.4235 & 35.1172 & 0.8126 & 0.4227 \\
\hline mean filter & 30.2667 & 5.5015 & 24.3765 & 35.0885 & 0.8291 & 0.5468 \\
\hline wiener filter & 19.7550 & 4.4447 & 26.2505 & 36.9414 & 0.8677 & 0.6335 \\
\hline proposed filter & 11.1425 & 3.3380 & 28.7395 & 39.4283 & 0.9329 & 0.7991 \\
\hline \multicolumn{7}{|c|}{ Grade 1 Placenta } \\
\hline & MSE & RMSE & SNR & $\begin{array}{l}\text { PSNR } \\
\end{array}$ & SSIM & UQI \\
\hline diffusion filter & 23.4549 & 4.8430 & 4.8430 & 35.7524 & 0.8602 & 0.4019 \\
\hline mean filter & 25.9858 & 5.0976 & 25.4537 & 35.3073 & 0.8736 & 0.5741 \\
\hline wiener filter & 13.0994 & 3.6193 & 28.4503 & 38.2822 & 0.9167 & 0.6502 \\
\hline proposed filter & 7.8416 & 2.8003 & 30.6802 & 40.5106 & 0.9561 & 0.7668 \\
\hline \multicolumn{7}{|c|}{ Grade 2 Placenta } \\
\hline & MSE & RMSE & SNR & PSNR & SSIM & UQI \\
\hline diffusion filter & 42.6805 & 6.5330 & 22.1915 & 32.5531 & 0.7860 & 0.4586 \\
\hline mean filter & 63.3604 & 7.9599 & 20.4313 & 30.8372 & 0.7494 & 0.4717 \\
\hline wiener filter & 36.9114 & 6.0755 & 22.8177 & 33.1838 & 0.8152 & 0.5792 \\
\hline proposed filter & 19.1188 & 4.3725 & 25.6826 & 36.0408 & 0.9195 & 0.8236 \\
\hline \multicolumn{7}{|c|}{ Grade 3 Placenta } \\
\hline & MSE & RMSE & SNR & PSNR & SSIM & UQI \\
\hline diffusion filter & 20.3646 & 4.5127 & 22.8805 & 35.4047 & 0.8757 & 0.4078 \\
\hline mean filter & 20.9073 & 4.5724 & 22.7414 & 35.2905 & 0.8895 & 0.5346 \\
\hline wiener filter & 10.8094 & 3.2878 & 25.6424 & 38.1555 & 0.9266 & 0.6403 \\
\hline proposed filter & 5.5919 & 2.3647 & 28.5127 & 41.0179 & 0.9671 & 0.8464 \\
\hline
\end{tabular}
LBP method and that of original image. The proposed method is also tested with ultrasound kidney images collected from ultrasound-images.com [36]. The quantitative results are shown in Table 2.

Table 1: MSE, RMSE, SNR, PSNR, SSIM, UQI values obtained for various filters 
Table 2: MSE, RMSE, SNR, PSNR, SSIM, UQI values for various filters applied on ultrasound kidney images

\begin{tabular}{|c|c|c|c|c|c|c|}
\hline & MSE & RMSE & SNR & PSNR & SSIM & UQI \\
\hline diffusion filter & 42.2944 & 6.5034 & 21.553 & 34.8783 & 0.8106 & 0.5223 \\
\hline mean filter & 72.9593 & 8.5416 & 19.1161 & 32.5103 & 0.7715 & 0.6543 \\
\hline wiener filter & 41.8041 & 6.4656 & 21.5932 & 34.9289 & 0.8283 & 0.7313 \\
\hline proposed filter & 21.2596 & 4.6108 & 24.5443 & 37.8655 & 0.9351 & 0.8975 \\
\hline
\end{tabular}

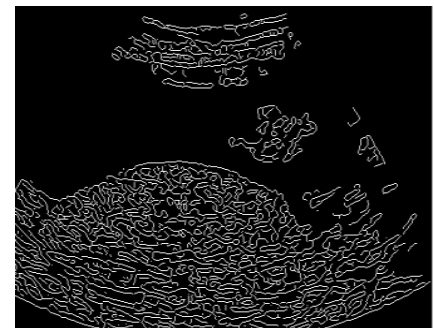

(a)

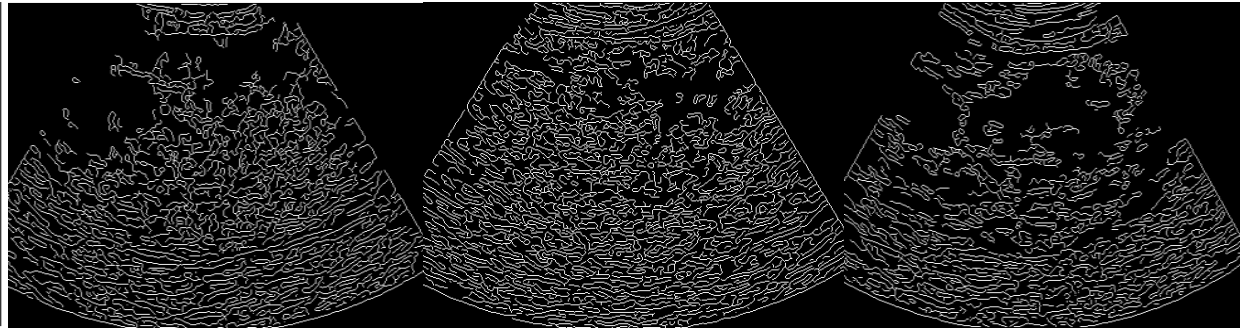

(b)

(c)

(d)

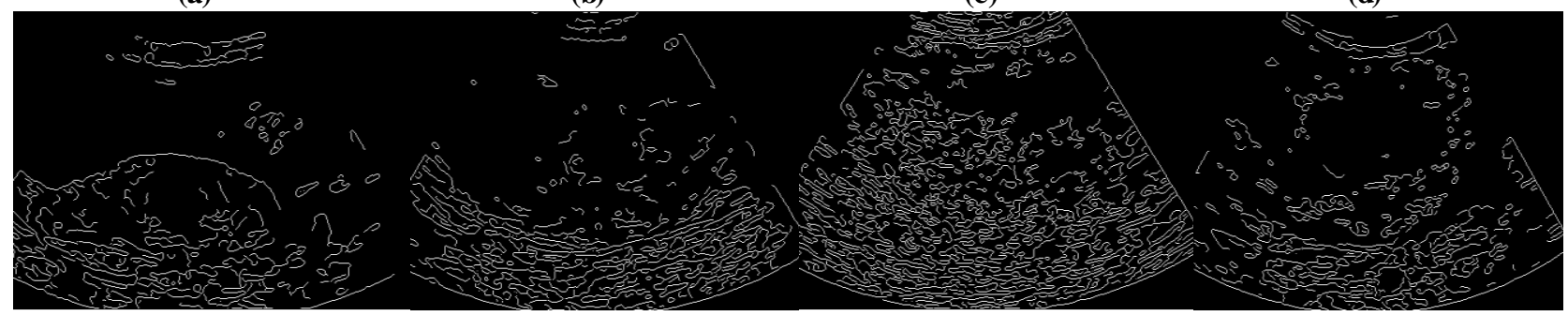

(e)

(f)

(g)

(h)

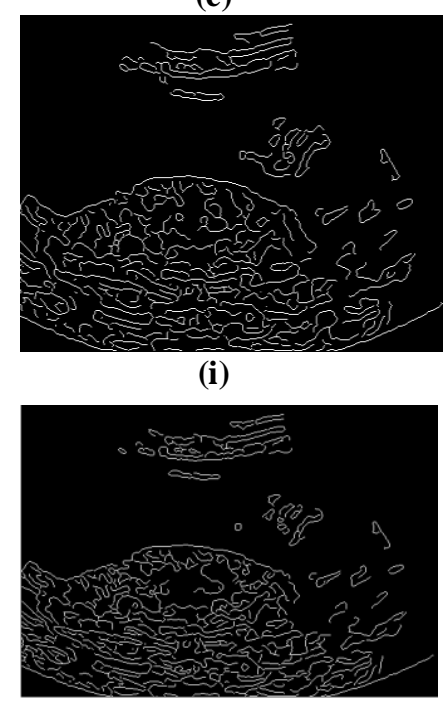

(m)

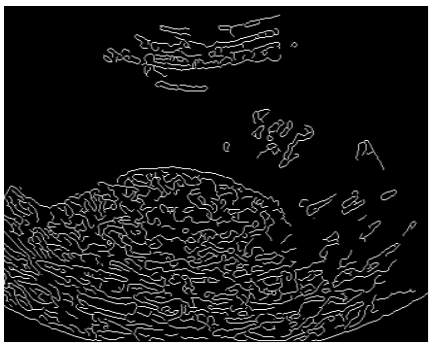

(q) (j)

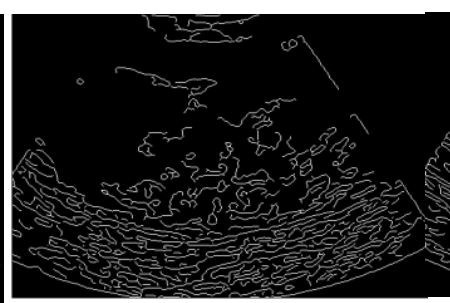

(n)

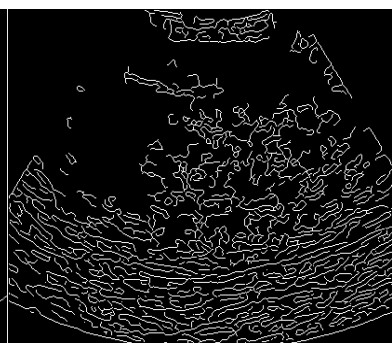

(r) (k)

(l) (p) (o)

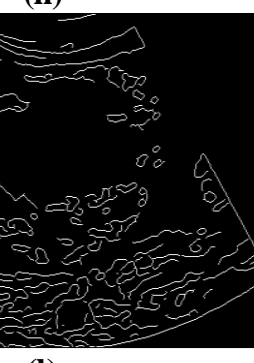




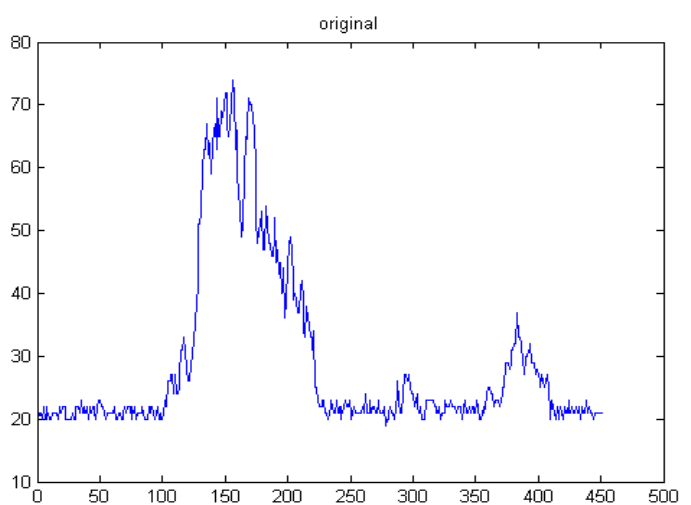

(a)

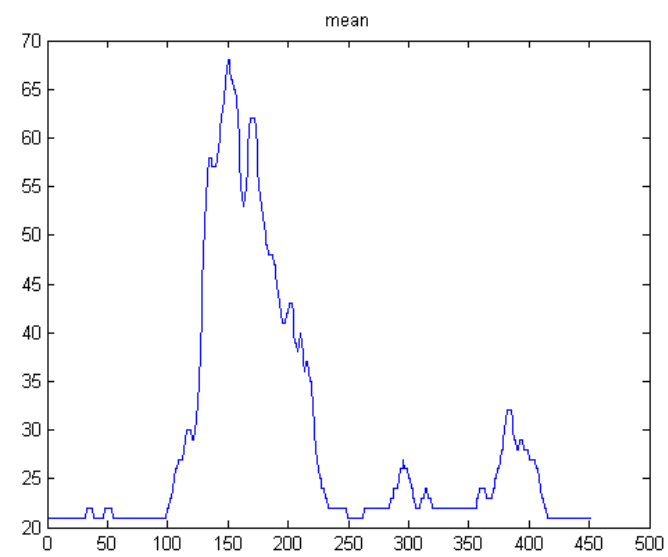

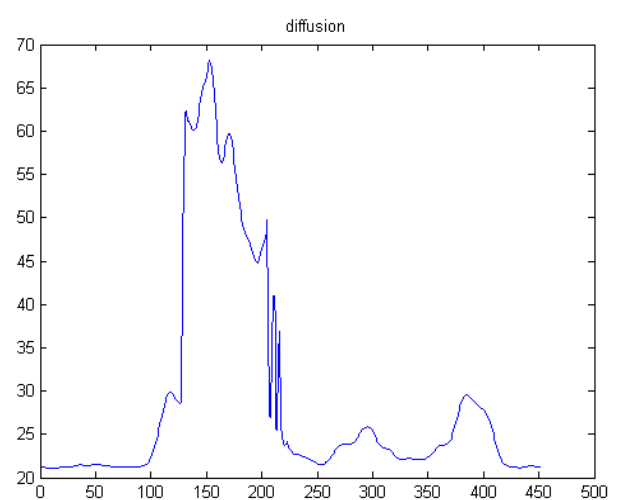

(b)

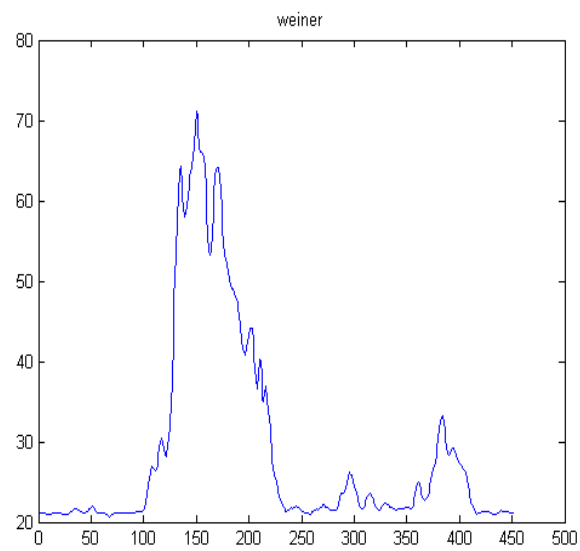

(d)

(c)

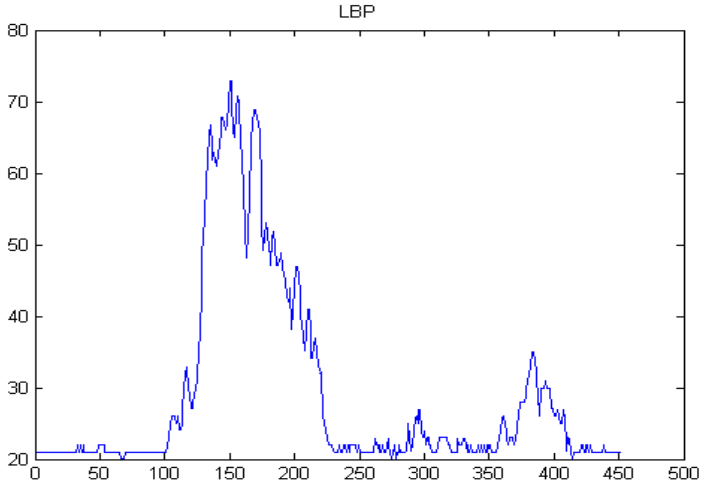

(e)

Fig. 4: intensity profile of a scan line through the center of the image-grade 1.(a) original image, (b-e) despeckled image using diffusion filter, mean filter, wiener filter and proposed LBP filter.

\section{Conclusion}

In this paper, we have developed a liner filter for reducing the speckle noise in ultrasound images. The filter is based on uniform local binary patterns of the image. Compared to other filters the proposed filter produces better result by reducing noise without affecting the relevant information. The quantitative measures and edge preservation of the proposed LBP method is high. The proposed method is tested with ultrasound images of placenta and kidney which are of great clinical importance.

\section{Reference}

[1] Zhang X, Smith N, Webb A, "Medical Imaging, Biomedical Information Technology", Academic 
Press Series in Biomedical Engineering, Elsevier, pp. 1-27,2008.

[2] Michailovich Oleg V, Tannenbaum A, "Despeckling of Medical Ultrasound Images", IEEE Transactions on Ultrasonics, Ferroelectrics, and Frequency Control, vol. 53, No.1, pp.64$78,2006$.

[3] Chinrungrueng C. Suvichakorn A., "Fast EdgePreserving Noise Reduction for Ultrasound Images", IEEE Transactions On Nuclear Science, vol.48, No. 3,pp. 849-854, 2001.

[4] Suvichakorn A, Chinrungrueng C, "Speckle Noise Reduction based on Least Squares Approximation", Proc. IEEE APCCAS, pp.430 - 433, 2000.

[5] Mohamed S, Mansoor Roomi, R.B.Jayanthi rajee, "Speckle Noise Removal In Ultrasound Images Using Particle Swarm Optimization technique”, Proc. ICRTIT, pp.926-931, 2011.

[6] Loupas T., Mcdicken W.N, Allan P.L, "An Adaptive Weighted Median Filter for Speckle Suppression in Medical Ultrasonic Images", IEEE Transactions On circuits and systems. Vol.36, No. 1,pp. 129-135. 1989.

[7] Sivakumar R, Gayathri M.K, Nedumaran D, "Speckle Filtering Of Ultrasound B-Scan Images A Comparative Study Between Spatial And Diffusion Filters",Proc. ICOS, pp.80-85, 2010.

[8] Ricardo G, Dantas, Eduardo T.C , "Ultrasound Speckle Reduction Using Modified Gabor Filters", IEEE Transactions on Ultrasonics, Ferroelectrics, and Frequency Control, vol. 54, no. 3, 530538 ,march 2007

[9] Gavriloaia G,Mariuca R.G, "Improving Quality of Medical Ultrasound Images by Filtering of Frames Sequences", Proc. HB 2011

[10] Xiaohui H, Shangkai G, Xiaorong G, “A Novel Multiscale Nonlinear Thresholding Method for Ultrasonic Speckle Suppressing”, IEEE Transactions On Medical Imaging, vol.18, No. 9,pp. 787-794,1999.

[11] Alin A, Anastasios B, P. Tsakalides," Novel Bayesian Multiscale Method for Speckle Removal in Medical Ultrasound Images", IEEE Transactions on Medical Imaging ,vol.20, No.8,pp. 772783,2001 .

[12] Rabbani H, Vafadust M, Abolmaesumi P, Saeed Gazor, "Speckle Noise Reduction of Medical Ultrasound Images in Complex Wavelet Domain Using Mixture Priors", IEEE Transactions On Biomedical Engineering, vol.55,N. 9,pp. 21522160,2008

[13] Pizurica A, Philips W,Lemahieu I, Marc Acheroy, "A Versatile Wavelet Domain Noise Filtration Technique for Medical Imaging”, IEEE
Transactions On Medical Imaging, vol.22, No. 3,pp.323-331,2003

[14] Gnanadurai D , Sadasivam V , Paul Tiburtius Nishandh , Muthukumaran L, Annamalai C, "Undecimated double density wavelet transform based speckle reduction in SAR images", Computers and Electrical Engineering vol.35 ,pp. 209-217,2009.

[15] Alka Vishwa, Shilpa Sharma, Modfied Method for Denoising the Ultrasound Images by wavelet Thresholding, I.J. Intelligent Systems and Applications, 2012, 6, 25-30

[16] Yongiian Yu ,Joseph Yadegar,” Regularized Speckle Reducing Anisotropic Diffusion For Feature Characterization", ICIP ,pp. 1577-1580, 2006.

[17] Ricardo G. Dantas, Eduardo T. Costa, "Ultrasound Speckle Reduction Using Modified Gabor Filters", IEEE transactions on ultrasonics, ferroelectrics, and frequency control, vol.54, No. 3,pp. 530-538, 2007.

[18] Hong-Ying Yang , Xiang-Yang Wanga, TianXiang Qua, Zhong-Kai Fu , "Image denoising using bilateral filter and Gaussian scale mixtures in shiftable complex directional pyramid domain" , Computers and Electrical Engineering vol.37 ,pp. 656-668,2011.

[19] Bhadauria H.S, M.L. Dewal, "Medical image denoising using adaptive fusion of curvelet transform and total variation ", Computers and Electrical Engineering, Article in press

[20] Abramowicz J.S, Sheiner E, "Ultrasound of the Placenta: A Systematic Approach. Part I: Imag ing", Placenta vol.29 ,pp. 225-240, 2008

[21] Loizou C.P, C.S. Pattich is , "Despeckle Filtering Algorithms and Software for Ultrasound Imaging", Morgan \& Claypool Publishers, CA, USA, 2008.

[22] Leonid Yaroslavsk, Dig ital Holography and Dig ital Image Processing:: Principles, Methods, Kluwer academic publishers, U.S.A, 2004

[23] Perona P, Malik J, Scale Space and Edge Detection Using anis otropic Diffusion, IEEE Transactions on Pattern Analysis and Machine Intelligence.12:7(1990).

[24] Klaus D. Toennies, Guide to Medical Image Analysis: Methods and Algorithms, Springer 2012.

[25] T. Ojala, M. Pietikäinen, T. Maenpaa, "Multiresolution Gray-scale and Rotation Invariant Texture Classification with local Binary patterns," IEEE Transactions on Pattern Analys is and Machine " Intelligence, vol.24 pp. 971-987,2002.

[26] Ahonen, T, Hadid, A,Pietikainen M, "Face Description with Local Binary Patterns: Application to Face Recognition”. IEEE Trans. 
Pattern Analysis and Machine Intelligence vol.28, No.12,pp. 2037-2041,2006.

[27] Hadid, A, Pietikainen, M, "Combining Appearance and Motion for Face and Gender Recognition from Videos". Pattern Recognition 42:11 pp. 28182827,2009.

[28] Baopu Li , Q.H.Max ,Meng, IEEE, “Tumor Recognition in Wireless Capsule Endoscopy Images Using Textural Features and SVM-Based Feature Selection", IEEE TRANSACTIONS ON INFORMATION TECHNOLOGY IN BIOMEDICINE, vol.16: 3,pp. 323-329,2012

[29] Malathi S, Meena C, “An efficient method for partial fingerprint recognition based on Local Binary Pattern”, Proc of ICCCCT'10, pp. 569-572.

[30] Han mandlu1 M, Ankit Gu reja, Ankur Jain, " Palm Print Recognition using Local Binary Pattern Operator and Support Vector Machines", Proc. ICSIP (2010) pp. 158-162

[31] SeyedMohsen Zabihi, Hamid Reza Pourreza, Touka Banaee, "Vessel Extraction of Conjunctival Images Using LBPs and ANFIS", ISRN Machine Vision, Vol. 2012, Article ID 424671.

[32] Maenpaa Topi, Ojala Timo, Pietikainen Matti, Soriano Maricor, "Robust Texture Classification by Subsets of Local Binary Patterns", Proc. ICPR, 935-938, 2000.

[33] Liao, S., Law M.W.K. , Chung A.C.S, ” Dominant Local Binary Patterns for Texture Classification". IEEE Trans. Image Processing 18:5,pp. 1107$1118,2009$.

[34] Tan X, Triggs B, "Fusing Gabor and LBP feature Sets for Kernel-based Face Recognition", Proc.International Workshop on Analysis and Modeling of Face and Gesture, 2007, pp.235-249.

[35] Heikkilä, M, Pietikäinen, M. “A Texture-Based Method for Modeling the Background and Detecting Moving Objects". IEEE Trans. Pattern Analysis and Machine Intelligence, vol.28(4) pp.657-662.,2006.

[36] http://www.ultrasound-images.com

\section{Authors' Profiles}

Simily Joseph: received her master degree in Computer Applications from M.G. University, Kerala, India in 2006. Presently she is a research scholar in the Dept. of Computer Applications, Cochin University of Science and Technology. She has several journal and conference publications in National and international level. Her research interest is in Medical Imaging, Content Based image Retrieval, Data Mining and Pattern Recognition.
M.R. Balachandran Nair: received his MBBS degree from Kottayam Medical College, University of Kerala. He completed his radiology residency at Armed Force Medical College Pune and Command Hospital, Calcutta. Currently he is working as a consultant radiologist. He served as a graded and classified specialist in Army and Navy. He has participated in various CME programmes conducted in Armed Forces.

Reji Rajan Varghese: received his MBBS degree from Kerala University in 2000 and M Tech in Biomedical Engineering from Vellore Institute of Technology in 2009. He also holds a post graduate diploma in ultrasonography. Presently he is working as senior resident in radiology and head of Dept. of Biomedical Engineering, Cochin Medical College. His areas of expertise are Biomedical Engineering, Medical Imaging, Radiology and Image Processing.

Kannan Balakrishnan: is an Associate professor in the Department of Computer Applications, Cochin University of Science and Technology, India. He took his $\mathrm{Ph} \mathrm{D}$. in Graph Algorithms from the Dept. of Futures Studies, University of Kerala. He has obtained M.sc and M.Phil in Mathematics from University of Kerala and M.Tech in Computer Science from Cochin University of Science and Technology. He is the co investigator of indo-Slovenian joint research project by Dept. of Science and Technology, Govt. of India. He has published several papers in international journals and national and international conference proceedings. $\mathrm{He}$ is a reviewer of American Mathematical Reviews. His areas of interest are Graph Algorithms, Intelligent systems, Image Processing, CBIR and Machine Translation.

How to cite this paper: Simily Joseph, Kannan Balakrishnan, M.R. Balachandran Nair, Reji Rajan Varghese,"Ultrasound Image Despeckling using Local Binary Pattern Weighted Linear Filtering", International Journal of Information Technology and Computer Science(IJITCS), vol.5, no.6, pp.19, 2013. DOI: $10.5815 /$ ijitcs.2013.06.01 